\title{
In silico Docking and 3D-QSAR Studies of Novel $N$ '-substituted-(pyrrolyl-phenoxy) Acetohydrazides as Enoyl-ACP Reductase Antagonists
}

\author{
Shrinivas Dattatraya Joshi, ${ }^{1, *}$, SR Prem Kumar ${ }^{1}$, Venkatarao H Kulkarni ${ }^{1}$, Ali Mohamed Alshabi ${ }^{2}$, \\ Ibrahim Ahmed Shaikh ${ }^{3}$, TM Aminabhavi' \\ ${ }^{1}$ Department of Pharmaceutical Chemistry, S.E.T's College of Pharmacy, Sangolli Rayanna Nagar, Dharwad, Karnataka, INDIA. \\ ${ }^{2}$ Department of Clinical Pharmacy, College of Pharmacy, Najran University, Najran, SAUDI ARABIA. \\ ${ }^{3}$ Department of Pharmacology, College of Pharmacy, Najran University, Najran, SAUDI ARABIA
}

\begin{abstract}
In search of potent anti-mycobacterial agents, enoyl-ACP reductase enzyme found to be most probable due to its imperative role in type II fatty acid synthesis (FAS), while mycolic acid production in Mycobacterium tuberculosis (M. tuberculosis), which is the appropriate entity for the discovery of antimycobacterial agents due to its primary role in the metabolism. Hence, blocking of enoyl ACP reductase would be important to develop novel antitubercular drugs development. Pyrrole, which is one of the five membered heterocyclic moieties habitually institute in few of naturally occurring and organic products of various classes, plays a significant part in medicinal drug discovery. In this work, docking and 3D-QSAR (CoMFA and CoMSIA) studies were performed on a set of pyrrolyl phenoxy acetohydrazides. As per docking study, TYR158 and co-factor NAD + are important amino acid residue at active site of enzyme for binding with the ligand. Among all the tested molecules, Compounds 4 and 28 have shown good docking scores of 7.08 and 8.85 respectively, steric and electrostatic field in CoMFA model displayed $q^{2}=0.511, r^{2}=0.822$ while that of CoMSIA model displayed $q^{2}=0.625, r^{2}=0.764$ with the SEE values of of 0.314 and 0.607 . Docking study revealed complete structurally imperative binding topographies between the pyrrole scaffolds and enoyl ACP reductase enzyme. Our studies offered useful information and evidence for designing of molecules with enhanced InhA inhibition.
\end{abstract}

Key words: Pyrrol-(1H-yl-phenoxy)acetohydrazides, Enoyl ACP reductase (InhA), Molecular Docking, 3D-QSAR, CoMFA, CoMSIA.

\section{INTRODUCTION}

Tuberculosis (TB) is one of the deadly bacterial diseases responsible for major universal deaths and caused by Mycobacterium tuberculosis (M. tuberculosis) strain, TB alone accounted for millions of deaths among adults in developing countries. Therefore, the infection often due to slow-growing mycobacteria recurrently exists in lungs (pulmonary TB) should be controlled. This contagious disease could spread to other parts of the body, referred to as extra-pulmonary TB which may be hidden or active. As per the recent World Health Organization (WHO) reports on TB, nearly 10 million people are infected by $M$. tuberculosis, worldwide. In 2017 alone 6.4 million new TB cases were officially reported to WHO, this shows number of new TB cases has been increasing since 2013. TB caused 1.3 million deaths among normal individuals and there were an extra 3,00,000 deaths from TB amongst HIV-positive individuals. Due increasing condition of drug resistance and multi drug resistance (XRD) by clinically important pathogens to available antibiotic therapy is of major alarm in medical civic. ${ }^{1}$ Antitubercular complexes which act precisely through altered
Submission Date: 12-10-2019; Revision Date: 25-06-2020; Accepted Date: 07-09-2020

DOI: 10.5530/ijper.54.3s.162 Correspondence: Dr. Shrinivas D. Joshi Novel Drug Design and Discovery Laboratory, Department of Pharmaceutical Chemistry, SET s College of Pharmacy, Sangolli Rayanna Nagar, Dharwad-580002, Karnataka, INDIA.

Phone: +91 9986151953

E-mail: shrinivasdj@ rediffmail.com

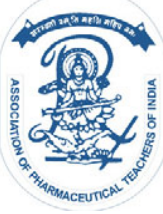

www.ijper.org 
mechanisms of actions from available medications would be recommend for better expectations to fight $\mathrm{XRD}-\mathrm{TB} .{ }^{2}$ InhA is one of the key enzyme necessary for the synthesis of fatty acid (FAS-II), which actively involved to yield $\mathrm{NAD}^{+}$and reduced enoyl thioesterACP substrate upon reduction of NADH-linked-trans 2-enoyl ACP, which is significant in metabolism of fatty acids and is also a beneficial studied target with different metabolic pathway plays a major role in antimicrobial drug discovery. In addition, bacterial ENR chain and basic skeletal association stay preferentially diverse when compare to human fatty acid biosynthetic (FAB) enzymes. ${ }^{3,4}$ In advancement of new scaffolds with antitubercular activity, we planned to inhibit the bacterial FAS-II system.

Pyrrole, being one of the important heterocyclic moiety present in many of the natural and synthetic derivatives with wide choice of pharmacological actions, such as antibacterial, ${ }^{5}$ antitubercular, ${ }^{6}$ antifungal, ${ }^{7}$ antiviral, ${ }^{8}$ antimicrobial ${ }^{9}$ and anti-inflammatory, ${ }^{10}$ since a large number of marketed medicines having the pyrrole structure. Even with its wide variety of pharmacological activities, not many studies have been studied against the TB related to other class of medicines. BM212 and LL3858 (phase II trial drug) are the known antitubercular drugs holding the pyrrole as core ring. Peptide linkages characterized by the existence of carbonyl linkage (-CO-NH-) is retained with synthesized compounds since, they are important for pharmacological actions like antibacterial, antiviral, anticancer and antitubercular properties. ${ }^{11-14}$

Molecular docking studies have proved that the structural topographies that are essential for binding of ligands with amino acids and $\mathrm{NAD}^{+}$co-factor existing in the binding pocket of InhA-enzyme active site. 3D-QSAR studies are also vibrant topics in design of drugs and discovery research to study drug-ligand approaches. However, 3D-QSAR analysis is a familiar scientific way of in-silico drug design tool. Amongst, CoMFAcomparative molecular field analysis was established by Cramer and his team ${ }^{15}$ has stood commonly quantified tool in drug discovery study, which provides steric and electrostatic regions for the biological activity prediction of molecules. Likewise, CoMSIA-comparative molecular similarity indices analysis models were employed to compute similarity indices at recurrently spaced grid points for the assigned moieties by using probe atom. The CoMSIA is diverges from those of CoMFA model, by the way in which calculations of molecular fields are done by Gaussian-type distance-dependent operation to quantity physicochemical properties, which includes steric, electrostatic, hydrophobic, hydrogen bond acceptor and donor fields. ${ }^{16,17}$

Literature suggest pyrroles heterocyclics as accountable antitubercular agents, they act as practical leads for the design and development of potent and broader spectrum antitubercular medications. In prior studies, we have described about docking analysis of pyrolyl hydrazones, ${ }^{1} \quad$ pyrrolyl-linked-1,3,4-oxadiazoles, phthlazines, pthalazinones, pyridazinones and pyrroleschiff base analogues. ${ }^{19-22}$ Further up taking of earlier reported studies, here in we state the docking analysis and 3D-QSAR investigation of pyrrole acetohydrazides ${ }^{18}$ as antimycobacterial moieties.

\section{MATERIALS AND METHODS}

\section{Data set}

Present study includes 53 active InhA inhibitors, molecules were taken from our own earlier stated data ${ }^{18}$ with definite MIC $(\mu \mathrm{g} / \mathrm{ml})$ values obtained for their in vitro antitubercular activity. The molecules under study was split into two different sets called training set (43 compounds) and a test set (10 compounds) by considering $80 \%$ of total molecules in the training set and $20 \%$ in the test set. Chemical diversity method was employed for building of training and test set used. Training set is necessary to design 3D-QSAR models, whereas quality of the produced model remained authenticated by means of test set. The reported molecular skeleton structure and activity information for the studied group of molecules are shown in Table 1.

\section{Molecular modeling and database alignment}

3D skeletal structures of pyrrole scaffolds were built using $^{23}$ molecular docking software SYBYL-X 2.0 by referring the standard geometric parameters. Practice of virtually low energy conformity in the arrangement is beneficial for the statistical assessment of flexible structures within CoMFA model. In present research, structure of all 53 pyrrole derivatives was geometrically enhanced by applying standard Tripos molecular mechanics force field with a distance-dependent $(1 / r)$ dielectric function and energy gradient convergence criterion of $0.05 \mathrm{kcal} / \mathrm{mol}$. Besides, requirement of partial atomic charges are needed for the assessment of electrostatic potential and are aligned using the Gasteiger-Huckel model. All the structures taken from the study were fitted into a lattice box by fitting with pyrrolyl phenoxy acetohydrazides basic structure taking compound $\mathbf{1 9}$ as a template. Figure 1 displays 


Table 1: Structures and antitubercular activity ex-

31 (1.6

All above molecules used for the study were taken from our own earlier data ref. Joshi et al..$^{18}$

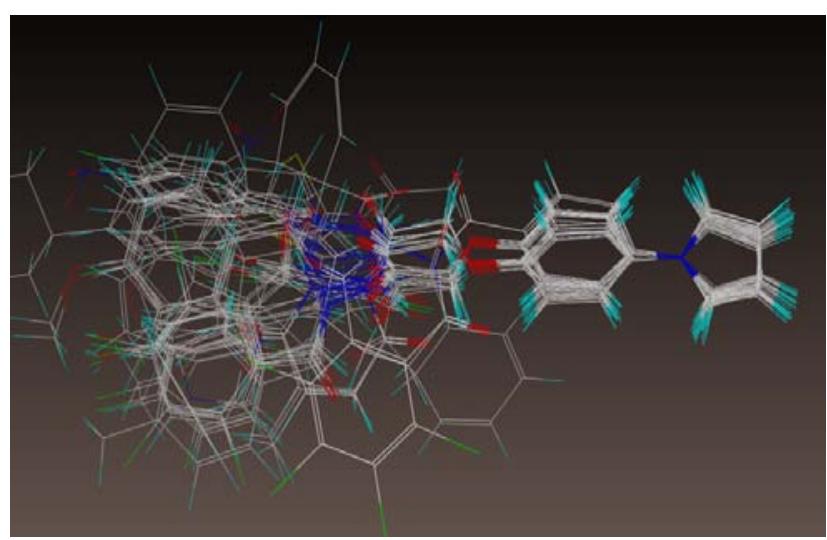

Figure 1: Database alignment of 53 compounds. 
the arrangement of all 53 pyrrole scaffolds referred in present work.

\section{Molecular docking}

Docking analysis was done on the crystal structure of M. tuberculosis InhA inhibited by triclosan were taken from the Protein Data Bank (PDB Code-2X22, 1.8 $\AA$, chain-A) by means of similar docking technique as defined earlier. ${ }^{18}$ Binding of pyrrole scaffolds was also projected through a range of scoring functions that have been collate into a specific consensus score (CScore).

\section{CoMFA and CoMSIA setup}

3D-QSAR techniques such as $\mathrm{CoMFA}^{24}$ and CoMSIA are the commonly used computational methods in the field of drug discovery area, ${ }^{15}$ since these QSAR models are proficient in predicting the biological activity of novel molecules by creating a relation between steric/ electrostatic properties and biological activities in the form of contour maps. In the present study, ligands are put in a 3D lattice and then steric as well as electrostatic fields of ligands were calculated at various grid points of the lattice. Partial Least Squares (PLS) was then studied to evaluate the resulting field matrix.

CoMSIA is one of the more current 3D-QSAR approaches with steric and electrostatic highlights. Separated from this, it too calculates H-bond donor, $\mathrm{H}$-bond acceptor and hydrophobic interactions. The CoMSIA likeness records properties were calculated employing a test with a span of $1.0 \AA$ and a default value of 0.3 as the attenuation figure. A grid spacing of $2 \AA$ was utilized for both CoMFA and CoMSIA. The values of $q^{2}$ and the lowest standard error of prediction (SEP) were calculated using:

$q^{2}=1-\frac{\sum_{y}\left(Y_{\text {pred }}-Y_{\text {actual }}\right)}{\sum_{y}\left(Y_{\text {pred }}-Y_{\text {actual }}\right)}$

where $Y_{\text {pred }}$ is the predicted activity, $Y_{\text {actual }}$ is the experimental activity and $\mathrm{Y}_{\text {mean }}$ is the best estimate of the mean.

$$
\mathrm{SEE}, \mathrm{SEP}=\sqrt{\frac{P R E S S}{n-C-1}}
$$

where $n$ is the number of compounds, $c$ is the number of components and PRESS is calculated as

PRESS $=\sum_{\mathrm{y}}\left(\mathrm{Y}_{\text {pred }}-\mathrm{Y}_{\text {actual }}\right)^{2}$

Similarity index, $\mathrm{A}_{F, k,}$ for a molecule, $j$ with atoms at the grid point, $q$ was calculated as:

$$
\mathrm{A}_{F, k(j)}^{\mathrm{q}}=-\sum \omega_{\text {probe, }} \omega_{\mathrm{ik}} \mathrm{e}^{-\alpha \gamma 2}{ }_{i q}
$$

where $\omega_{\text {probe, }}$ is the probe atom with radius $1 \AA$, charge +1 , hydrophobicity +1 , H-bond donating +1 and H-bond accepting $+1 ; \omega_{i k}$ is the actual value of physicochemical property $k$ of atom i; $\mathrm{r}_{\mathrm{iq}}$ is the mutual distance between the probe atom at grid point, $q$ and atom, $I$ of the test molecule; $\alpha$ is the attenuation factor, while the default value is taken as 0.3 . Five physicochemical properties, $k$ (steric, electrostatic, hydrophobic, H-bond acceptor and donor) were evaluated using a common charged, hydrophobic and H-bond accepting probe atom.

\section{PLS analysis}

PLS study will offers an exact association between antitubercular activity of molecules with that of the projecting values of CoMFA and CoMSIA comprising the magnitude of steric, electrostatic, hydrophobic potentials, H-bond donor and acceptor. This partial least square regression analysis protocol was used to investigate the training set by parallel comparison of dependent variables (antitubercular activity) along with the dissimilarities in their independent variables (CoMFA/CoMSIA interaction fields). Leave one out (LOO) stratergy and Samples-distance Partial Least Square (SAMPLS) was utilized to decide the ideal number of components driving to the highest cross-validated coefficient $q^{2} \cdot{ }^{25}$ SEP demonstrates the consistency and prescient capacity of the created models. After this, the ultimate PLS relapse examination was gotten from non cross validation method with the clarified distinction $r^{2}$, standard error of estimate (SEE) and $F$ ratio. The SEE decides the target unlikelihood properties however unexplained after the demonstrated model has been inferred and $F$ is the ratio of $r^{2}$ to $1-r^{2}$ weighted such that less the informative properties and more the values of the target property, higher will be the F-ratio. Prototype with an ideal number of components (highest $q^{2}$ ) and with the most reduced SEP was chosen for advanced analysis.

The bootstrapping procedure ${ }^{25}$ was utilized to approve each model. In this method, $n$ numbers of arbitrary choices out of the training set of $n$ objects were made a number of periods to duplicate a diverse sampling from bigger set of objects. In each trial, a few objects might not have been involved with in the PLS investigation and a few may have been comprised more than once.

\section{RESULTS AND DISCUSSION Molecular docking analysis}

Docking analysis of all 53 molecules was reported before on 4TZK ligand. ${ }^{18}$ Now we docked on the 2X22 $\mathrm{PDB}$ and the results of docked molecules are displayed in Figure 2A and 2B, while projected binding scores of the molecules are shown in Table 2. Triclosan (ligand) has shown a total docking score of 13.70. As depicted in Figures $3 \mathrm{~A}$ and $3 \mathrm{~B}$, the $-\mathrm{OH}$ group of triclosan makes two $\mathrm{H}$-bonding connections at dynamic site of 


\begin{tabular}{|c|c|c|c|c|c|c|c|}
\hline Compd. & C score ${ }^{a}$ & $\begin{array}{l}\text { Crash } \\
\text { score }^{\mathrm{b}}\end{array}$ & $\begin{array}{l}\text { Polar } \\
\text { score }^{\mathrm{c}}\end{array}$ & D score ${ }^{d}$ & PMF score ${ }^{e}$ & G score f $^{f}$ & Chemscore $^{g}$ \\
\hline $\begin{array}{l}2 \times 22 \\
\text { Ligand }\end{array}$ & 13.70 & -1.08 & 2.21 & -171.007 & -44.459 & -321.501 & -47.452 \\
\hline 29 & 9.20 & -1.81 & 0.00 & -169.132 & -54.755 & -331.890 & -38.230 \\
\hline 45 & 9.00 & -1.58 & 0.00 & -154.364 & -56.605 & -285.886 & -37.743 \\
\hline 28 & 8.85 & -1.49 & 2.06 & -168.348 & -64.311 & -255.995 & -40.017 \\
\hline 37 & 8.76 & -2.46 & 0.00 & -176.083 & -62.550 & -333.785 & -44.581 \\
\hline 21 & 8.48 & -0.99 & 0.13 & -173.486 & -75.142 & -257.239 & -35.858 \\
\hline 43 & 8.37 & -1.56 & 0.35 & -148.702 & -58.891 & -269.116 & -37.506 \\
\hline 19 & 8.14 & -1.51 & 0.35 & -136.561 & -56.824 & -267.304 & -36.398 \\
\hline 39 & 8.13 & -1.16 & 0.03 & -176.980 & -70.587 & -273.222 & -35.514 \\
\hline 20 & 8.10 & -1.65 & 0.23 & -142.157 & -63.221 & -279.108 & -37.877 \\
\hline 34 & 8.08 & -2.38 & 0.00 & -166.604 & -53.623 & -295.826 & -39.272 \\
\hline 44 & 7.95 & -1.81 & 0.00 & -144.754 & -72.459 & -268.408 & -36.781 \\
\hline 32 & 7.94 & -0.86 & 0.00 & -149.262 & -63.155 & -273.618 & -39.850 \\
\hline 18 & 7.90 & -1.87 & 0.00 & -145.768 & -62.449 & -267.112 & -29.689 \\
\hline 25 & 7.82 & -1.69 & 0.00 & -151.586 & -62.507 & -291.133 & -28.814 \\
\hline 40 & 7.77 & -1.31 & 0.00 & -152.971 & -58.478 & -268.649 & -39.572 \\
\hline 41 & 7.76 & -1.21 & 0.00 & -146.179 & -59.276 & -271.907 & -39.067 \\
\hline 31 & 7.72 & -1.51 & 0.00 & -148.316 & -54.848 & -280.021 & -36.951 \\
\hline 26 & 7.72 & -1.95 & 0.51 & -145.486 & -39.460 & -262.841 & -37.964 \\
\hline 35 & 7.58 & -1.18 & 0.00 & -152.963 & -49.641 & -297.304 & -39.350 \\
\hline 22 & 7.55 & -1.92 & 0.00 & -150.037 & -54.717 & -285.492 & -38.211 \\
\hline 42 & 7.46 & -1.23 & 0.00 & -146.663 & -74.719 & -276.959 & -38.131 \\
\hline 27 & 7.41 & -1.60 & 0.00 & -143.944 & -56.482 & -276.467 & -35.445 \\
\hline 23 & 7.33 & -1.33 & 0.00 & -147.608 & -43648 & -280.161 & -38.456 \\
\hline 16 & 7.332 & -1.52 & 0.00 & -167.660 & -80.573 & -312.316 & -37.958 \\
\hline 47 & 7.31 & -1.86 & 0.00 & -118.675 & -63.421 & -263.332 & -22.568 \\
\hline 33 & 7.31 & -1.85 & 0.00 & -168.760 & -58.631 & -277.658 & -37.212 \\
\hline 36 & 7.30 & -1.58 & 0.00 & -143.811 & -49.923 & -263.919 & -36.271 \\
\hline 10 & 7.29 & -1.72 & 0.01 & -179.484 & -81.761 & -279.869 & -33.939 \\
\hline 30 & 7.25 & -1.95 & 0.01 & -137.660 & -62.271 & -305.671 & -37.801 \\
\hline 02 & 7.19 & -0.78 & 0.00 & -132.142 & -71.326 & -253.921 & -27.959 \\
\hline 17 & 7.16 & -2.54 & 0.00 & -152.706 & -52.023 & -308.515 & -32.335 \\
\hline 38 & 7.15 & -1.62 & 0.00 & -141.355 & -58.160 & -262.431 & -36.663 \\
\hline 13 & 7.15 & -1.04 & 1.07 & -158.154 & -84.894 & -225.733 & -32.540 \\
\hline 46 & 7.13 & -0.92 & 0.57 & -118.532 & -47.243 & -246.383 & -24.824 \\
\hline 04 & 7.08 & -2.11 & 0.14 & -165.961 & -57.02 & -273.898 & -32.950 \\
\hline 11 & 6.98 & -0.87 & 0.00 & -138.750 & -66.932 & -248.577 & -27.698 \\
\hline 24 & 6.88 & -1.49 & 0.00 & -151.591 & -68.710 & -288.823 & -39.369 \\
\hline 12 & 6.82 & -1.13 & 0.00 & -135.018 & -67.353 & -252.926 & -27.461 \\
\hline 08 & 6.78 & -2.85 & 0.00 & -147.691 & -59.426 & -307.382 & -33.883 \\
\hline 01 & 6.63 & -1.51 & 0.00 & -151.635 & -79.689 & -271.426 & -35.197 \\
\hline 07 & 6.60 & -2.32 & 0.00 & -157.005 & -69.547 & -304.300 & -36.377 \\
\hline 48 & 6.55 & -2.18 & 0.00 & -171.144 & -77.453 & -311.191 & -37.802 \\
\hline 05 & 6.54 & -1.87 & 0.00 & -130.780 & -71.836 & -275.248 & -27.750 \\
\hline 03 & 6.52 & -1.43 & 0.00 & -138.042 & -69.199 & -269.931 & -29.871 \\
\hline
\end{tabular}




\begin{tabular}{|l|l|l|l|l|l|l|l|}
\hline $\mathbf{1 4}$ & 6.46 & -1.01 & 0.00 & -134.354 & -61.141 & -247.329 & -27.107 \\
\hline $\mathbf{0 9}$ & 6.39 & -1.66 & 0.00 & -138.396 & -60.955 & -275.005 & -28.620 \\
\hline $\mathbf{0 6}$ & 6.31 & -2.44 & 0.00 & -161.625 & -70.172 & -302.647 & -36.730 \\
\hline $\mathbf{5 0}$ & 5.54 & -2.46 & 0.00 & -128.584 & -72.528 & -259.468 & -31.652 \\
\hline $\mathbf{5 3}$ & 5.54 & -2.46 & 0.00 & -128.584 & -72.528 & -259.468 & -31.652 \\
\hline $\mathbf{5 2}$ & 4.16 & -1.57 & 0.00 & -125.433 & -76.233 & -218.013 & -38.364 \\
\hline $\mathbf{4 9}$ & 4.16 & -1.57 & 0.00 & -125.433 & -76.233 & -218.013 & -38.364 \\
\hline $\mathbf{1 5}$ & 2.44 & -1.54 & 0.00 & -116.920 & -58.127 & -187.821 & -34.966 \\
\hline $\mathbf{5 1}$ & 2.44 & -1.54 & 0.00 & -116.920 & -58.127 & -187.821 & -34.966 \\
\hline
\end{tabular}

${ }^{2} \mathrm{CScore}$ (Consensus Score) integrates a number of popular scoring functions for ranking the affinity of ligands bound to the active site of a receptor and reports the output of total score.

${ }^{b}$ Crash-score revealing the inappropriate penetration into the binding site. Crash scores close to o are favorable. Negative numbers indicate penetration.

'Polar indicating the contribution of polar interactions to the total score.

${ }^{\mathrm{d} D} \mathrm{D}$-score for charge and van der Waals interactions between the protein and the ligand.

ePMF-score indicating Helmholtz free energies of interactions for protein-ligand atom pairs (Potential of Mean Force, PMF).

${ }^{\mathrm{f}} \mathrm{G}$-score showing hydrogen bonding, complex (ligand-protein) and internal (ligand-ligand) energies.

${ }^{9} \mathrm{Chem}$-score points for $\mathrm{H}$-bonding, lipophilic contact and rotational entropy along with an intercept term.

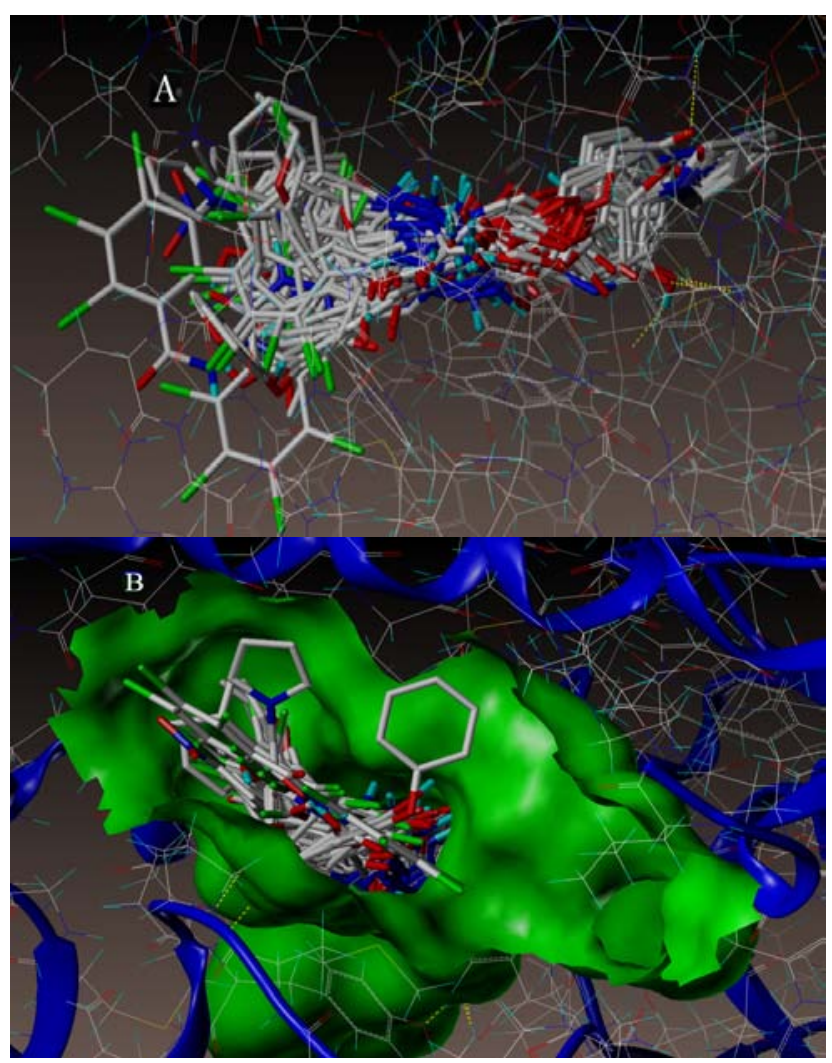

Figure 2: (A) All the molecules are docked into the active site of the enzyme (PDB 2X22); (B) Inside the proposed binding pocket of InhA.

the enzyme, $\mathrm{H}$-atom of -OH group bonds with oxygen of Tyr158 (OH----O-TYR158, $1.86 \AA)$ and oxygen of -OH group displayed bonding with hydrogen atom of NAD1270 (HO----H-NAD1270, $1.92 \AA)$. Figures 4 and 5 show the docking of potent molecules 4 and 28 into the active location of InhA enzyme. As represented in Figure 4, molecule 4 makes two H-bonding exchanges at the active place of the ENR enzyme, oxygen atom of

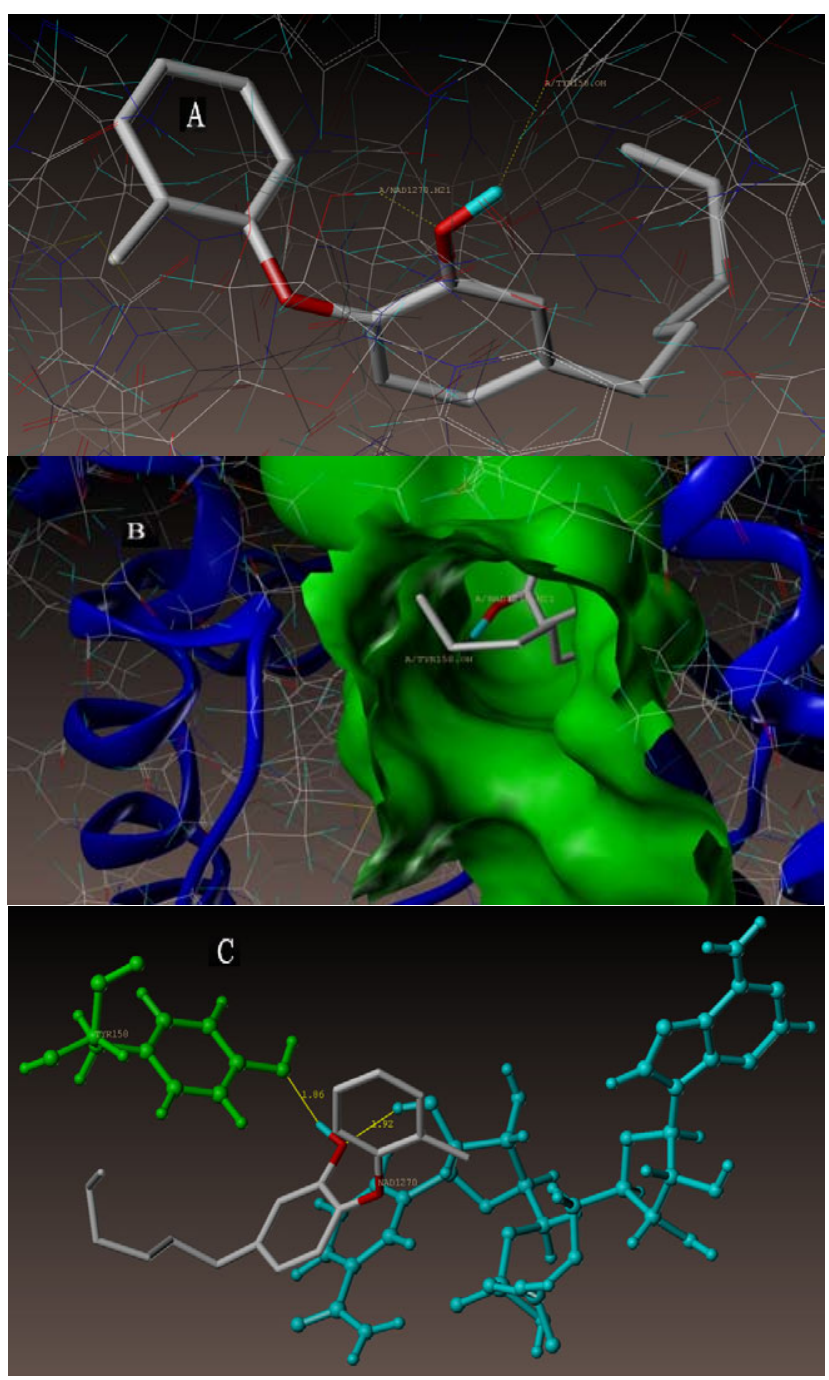

Figure 3: (A) Interaction of triclosan with crystal structure of enzyme (PDB 2X22); (B) Inside the proposed binding pocket of InhA and (C) 3D docked view of the 2X22_ligand. Binding site residues; green colored Tyr158 amino acid, cyan colored co-factor NAD+ and the molecule is colored according to atom type. 


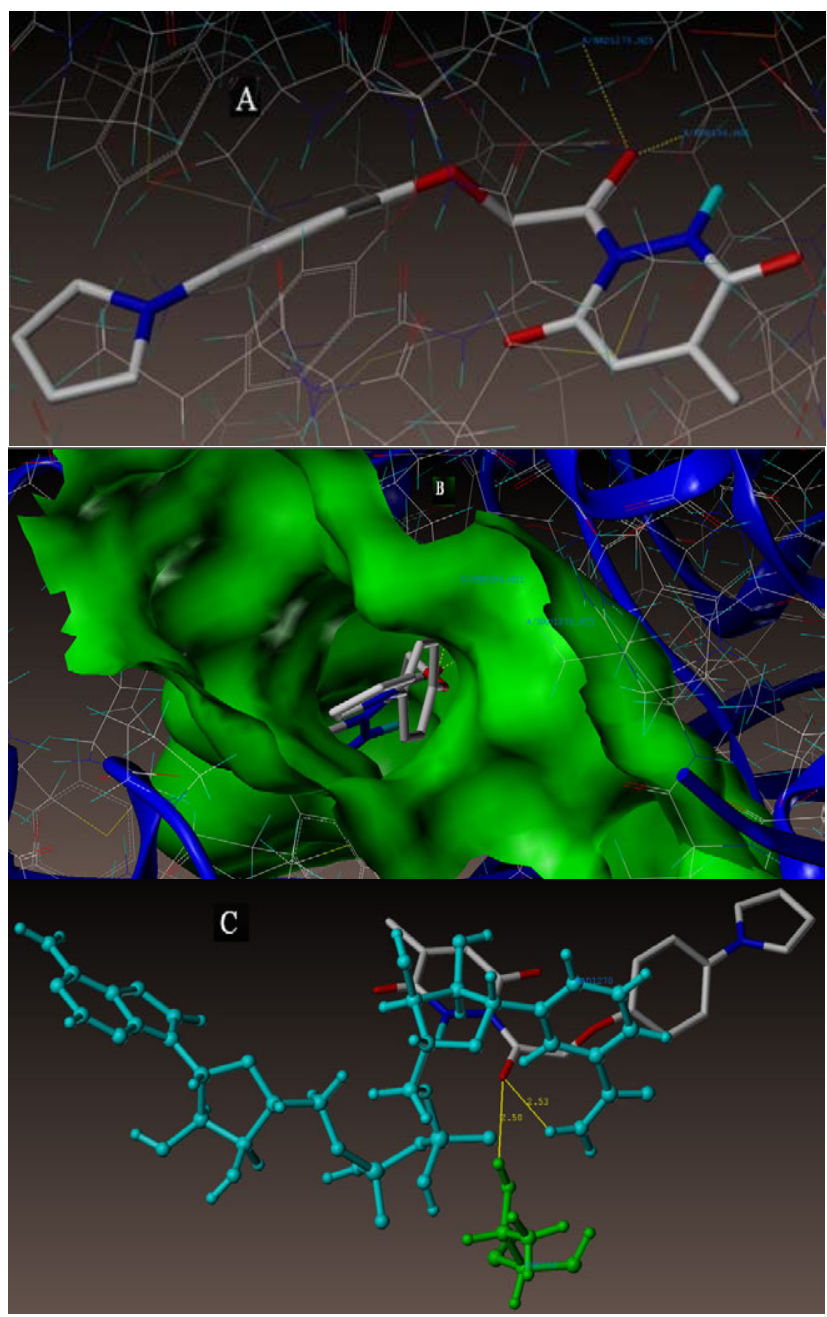

Figure 4: (A) Interaction of compound 4 with the crystal structure of enzyme (PDB 2X22); (B) Inside the proposed binding pocket of InhA and (C) 3D docked view of the 2X22_ligand. Binding site residues; green colored Thr 196 amino acid, cyan colored co-factor NAD+ and the molecule is colored according to atom type.

-CO group makes couple of H-bonding interactions with the hydrogens of NAD1270 (C=O----H-NAD1270, $2.53 \AA)$ and THR196 (C=O----H-THR196, $2.50 \AA)$. Compound 28 (Figure 5) showed two interactions, hydrogen atom of -OH group makes an interaction with oxygen atom of Tyr158 (OH----O-TYR158, 1.81 $\AA)$ and oxygen atom of hydroxyl group showed strong interaction with hydrogen atom of NAD1270 (HO---H-NAD1270, $1.83 \AA$ ).The amino acids [ARG173, ASN172, ASN187, GLN214, GLU169, GLU209, GLU210, NAD1270, SER186, SER200, THR253, TYR259, TYR158] and [ALA176, ALA190, ALA198, ALA201, ALA211, GLY96, GLY204, GLY205, ILE251, LEU188, MET147, MET199, VAL203] surrounded by the fragment of molecules are necessary to exhibit hydrophilic and hydrophobic communications thereby enhancing the potency of the compounds (Figures 6
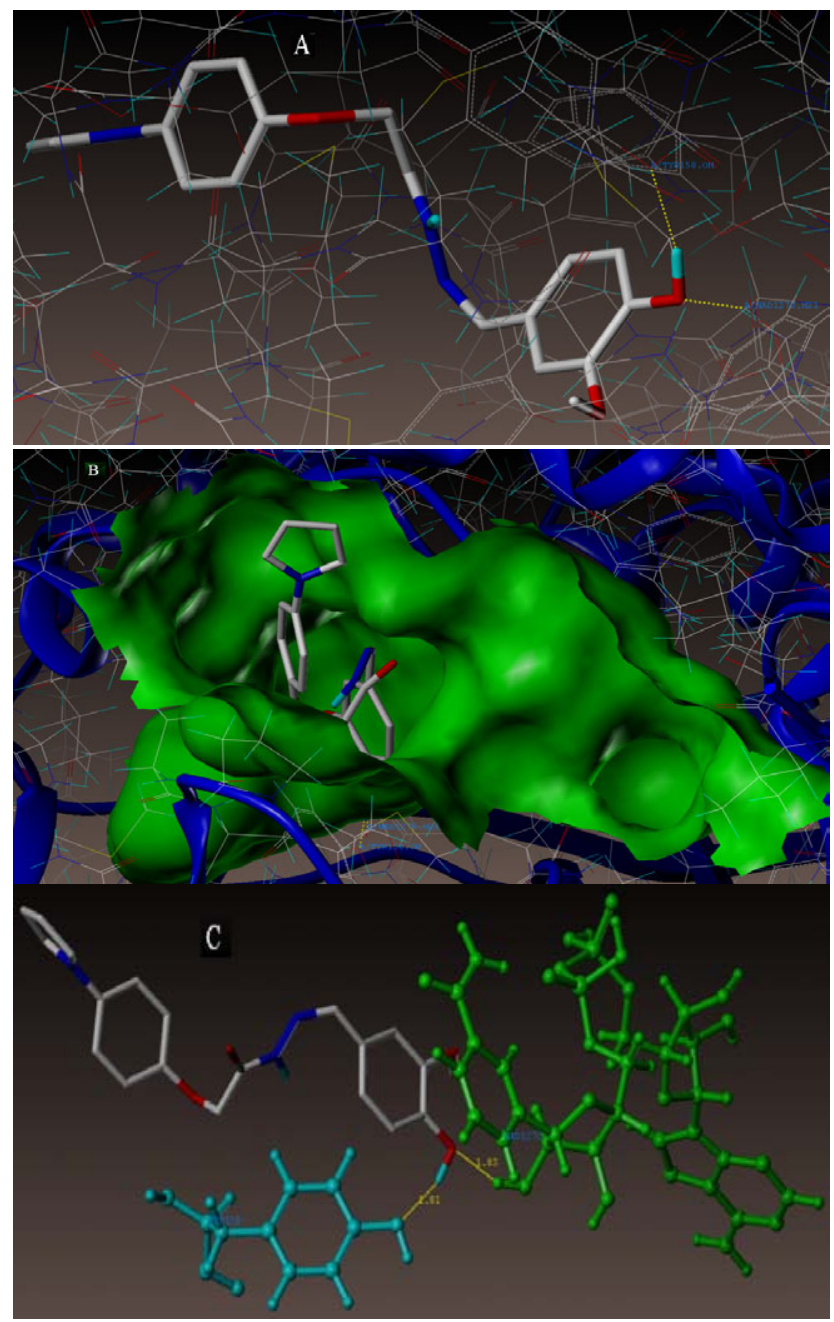

Figure 5: (A) Interaction of compound 28 with crystal structure of enzyme (PDB 2X22); (B) Inside the proposed binding pocket of InhA and (C) 3D docked view of the 2X22_ligand. Binding site residues; cyan colored Tyr 158 amino acid, green colored co-factor NAD+ and the molecule is colored according to atom type.

and 7). From docking values, it has been discovered that the molecules exhibited a good interface with an active site of enzyme similar to triclosan.

\section{D QSAR}

3D-QSAR study of 53 enoyl ACP reductase inhibitors were studied using CoMFA and CoMSIA techniques, the MIC values of stated molecules differ in the range of $0.2 \mu \mathrm{g} / \mathrm{ml}$ to $50 \mu \mathrm{g} / \mathrm{ml}$ (Table 1). Conforming in vitro (MIC) values were transformed to coordinating predictive-MIC (pMIC, -log MIC) standards, but then development of consistent representations is reliant on building of suitable test and training sets. Data of 53 inhibiting agents was splits into training groups (43 molecules) and test groups (10 molecules) (Table 1). Prescient capacity of the created replicas was appeared in Table 3. 


\begin{tabular}{|c|c|c|c|c|c|c|c|c|c|c|c|}
\hline \multirow[b]{2}{*}{ Compd. } & \multirow{2}{*}{$\begin{array}{l}\text { Actual } \\
\text { pMIC }\end{array}$} & \multicolumn{2}{|c|}{ CoMFA } & \multicolumn{2}{|c|}{ CoMSIA } & \multirow[b]{2}{*}{ Compd. } & \multirow{2}{*}{$\begin{array}{l}\text { Actual } \\
\text { pMIC }\end{array}$} & \multicolumn{2}{|c|}{ CoMFA } & \multicolumn{2}{|c|}{ CoMSIA } \\
\hline & & $\begin{array}{l}\text { Predicted } \\
\text { pMIC }\end{array}$ & $\Delta^{\mathrm{a}}$ & $\begin{array}{l}\text { Predicted } \\
\text { pMIC }\end{array}$ & $\Delta^{\mathrm{a}}$ & & & $\begin{array}{l}\text { Predicted } \\
\text { pMIC }\end{array}$ & $\Delta^{\mathrm{a}}$ & $\begin{array}{l}\text { Predicted } \\
\text { pMIC }\end{array}$ & $\Delta^{\mathrm{a}}$ \\
\hline 1 & 5.79 & 5.69 & 0.10 & 5.48 & 0.31 & 28 & 4.60 & 4.83 & -0.22 & 4.77 & -0.17 \\
\hline 2 & 5.79 & 5.68 & 0.10 & 5.71 & 0.08 & 29 & 5.20 & 5.00 & 0.20 & 4.79 & 0.40 \\
\hline 3 & 5.79 & 5.66 & 0.12 & 5.61 & 0.17 & 30 & 4.00 & 3.90 & 0.09 & 4.09 & -0.09 \\
\hline 4 & 5.79 & 5.62 & 0.17 & 5.56 & 0.23 & 31 & 5.79 & 5.75 & 0.03 & 4.79 & 1.00 \\
\hline 5 & 5.79 & 5.85 & -0.06 & 5.57 & 0.22 & 32 & 4.60 & 4.62 & -0.02 & 4.15 & 0.44 \\
\hline 6 & 4.30 & 4.47 & -0.17 & 5.40 & -1.10 & 33 & 4.60 & 4.45 & 0.14 & 4.53 & 0.06 \\
\hline 7 & 4.30 & 4.39 & -0.09 & 5.41 & -1.11 & 34 & 5.79 & 5.78 & 0.01 & 4.74 & 1.05 \\
\hline $8^{*}$ & 5.79 & 6.03 & -0.23 & 6.09 & -0.30 & 35 & 4.30 & 4.33 & -0.02 & 4.70 & -0.40 \\
\hline 9 & 5.79 & 6.02 & -0.23 & 5.62 & 0.17 & 36 & 4.90 & 4.94 & -0.04 & 4.76 & 0.14 \\
\hline $10^{*}$ & 5.79 & 6.00 & -0.20 & 5.46 & 0.32 & $37^{*}$ & 5.50 & 5.42 & 0.08 & 5.98 & -0.48 \\
\hline 11 & 4.30 & 4.98 & -0.67 & 4.95 & -0.35 & 38 & 4.30 & 4.26 & 0.03 & 4.72 & -0.42 \\
\hline 12 & 5.79 & 5.20 & 0.58 & 4.95 & 0.34 & 39 & 4.30 & 3.97 & 0.33 & 4.75 & -0.45 \\
\hline 13 & 4.30 & 4.54 & -0.24 & 4.44 & -0.14 & $40^{*}$ & 5.50 & 5.32 & 0.18 & 5.43 & 0.07 \\
\hline 14 & 4.30 & 4.61 & -0.31 & 4.59 & -0.29 & 41 & 5.79 & 5.11 & 0.68 & 5.18 & 0.50 \\
\hline $15^{*}$ & 4.30 & 4.42 & -0.12 & 4.62 & -0.32 & 42 & 5.79 & 5.27 & 0.52 & 4.90 & 0.88 \\
\hline 16 & 4.30 & 4.26 & 0.03 & 4.29 & 0.01 & 43 & 4.30 & 5.08 & -0.78 & 5.17 & -0.36 \\
\hline 17 & 5.79 & 5.95 & -0.16 & 5.49 & 0.30 & 44 & 5.79 & 5.76 & 0.03 & 5.25 & 0.54 \\
\hline 18 & 5.79 & 5.34 & 0.44 & 4.53 & 1.25 & 45 & 4.90 & 5.29 & -0.39 & 4.88 & 0.02 \\
\hline 19 & 4.00 & 3.95 & 0.05 & 4.58 & -0.51 & $46^{*}$ & 5.78 & 5.69 & 0.09 & 5.48 & 0.30 \\
\hline 20 & 4.30 & 4.52 & -0.24 & 4.67 & -0.37 & $47^{*}$ & 5.79 & 5.76 & 0.03 & 5.28 & 0.51 \\
\hline 21 & 4.90 & 4.77 & 0.15 & 4.91 & -0.01 & 48 & 4.30 & 4.08 & 0.21 & 4.16 & 0.13 \\
\hline 22 & 4.30 & 4.04 & 0.25 & 4.58 & -0.28 & 49 & 4.30 & 4.43 & -0.13 & 4.91 & -0.60 \\
\hline 23 & 4.30 & 4.72 & -0.46 & 4.68 & -0.38 & 50 & 5.79 & 5.64 & 0.15 & 5.27 & 0.51 \\
\hline $24^{*}$ & 4.30 & 4.34 & -0.04 & 4.87 & -0.57 & 51 & 4.30 & 4.44 & -0.13 & 4.88 & -0.58 \\
\hline 25 & 4.30 & 4.06 & 0.23 & 4.58 & -0.28 & $52^{*}$ & 4.30 & 4.34 & -0.04 & 4.63 & -0.33 \\
\hline 26 & 4.60 & 4.72 & -0.12 & 4.67 & -0.07 & $53^{*}$ & 5.79 & 5.53 & 0.26 & 5.00 & 0.79 \\
\hline 27 & 4.30 & 4.45 & -0.15 & 4.83 & -0.53 & & & & & & \\
\hline
\end{tabular}

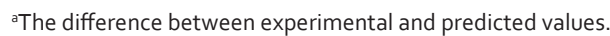

(* indicates test set)

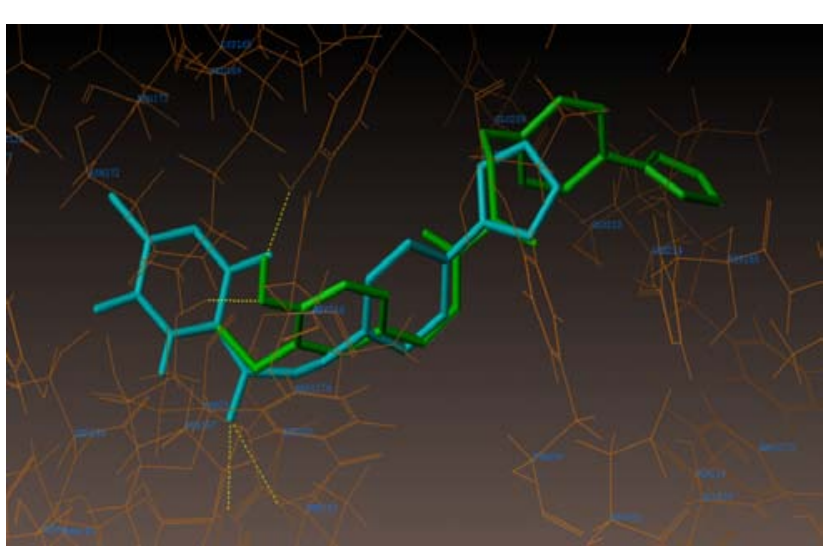

Figure 6: Hydrophiliclnteraction of compounds 4 and 28 with the crystal structure of enzyme PDB 2X22.

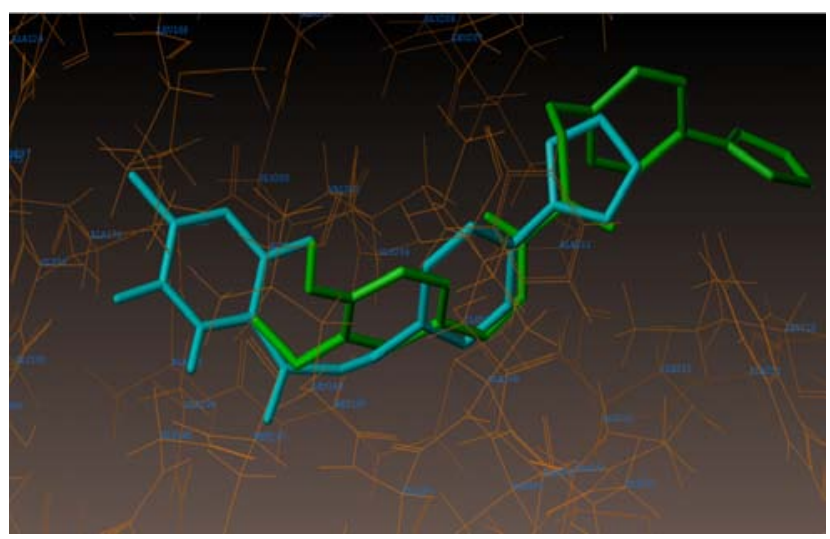

Figure 7: Hydrophobic Interaction of compounds 4 and 28 with the crystal structure of the enzyme PDB 2 X22. 
PLS strategy was utilized to construct individual 3D-QSAR representations. CoMFA and CoMSIA were considered as free variable whereas pMIC values as subordinate factors which are imperative in PLS relapse thinks about to development QSAR models. Models analytical capability was evaluated by their $q^{2}$ values (Table 4).

\section{CoMFA and CoMSIA model}

Upgraded version of molecular designing tool SYBYL-X 2.0 was used to perform CoMFA and CoMSIA experiments, atomic charges molecules were computed by Gasteiger-Huckel established rules. Statistical specifications gained after that of CoMFA and CoMSIA investigations was presented in Table 4. The superlative estimates gained for CoMFA prototype were established with $q^{2}=0.511\left(r^{2}=0.822\right.$, SEE $=$ $0.314, F=243.23)$ and for CoMSIA replica, $q^{2}=0.625$ $\left(r^{2}=0.764, S E E=0.607, F=177.36\right)$ for the particular requisite method. Predictive values for training and test sets from CoMFA and CoMSIA studies are presented respectively in Table 3. Figure 8, portrays the graphs
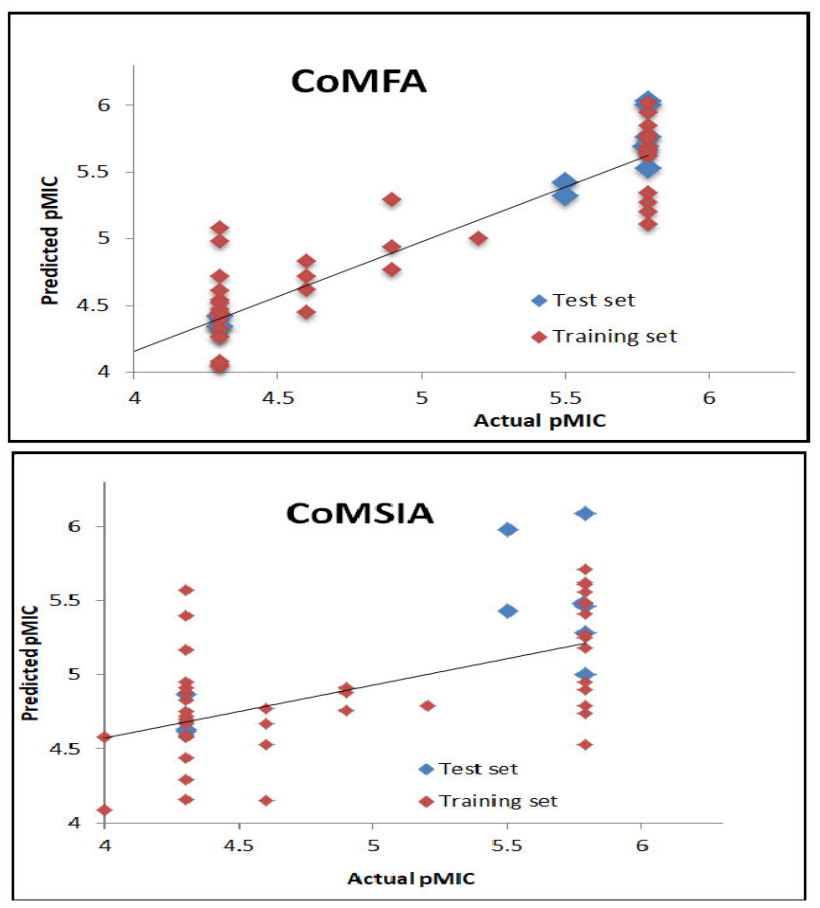

Figure 8: Scatter plot diagram for (A) CoMFA and (B) CoMSIA analyses by database alignment.

\begin{tabular}{|c|c|c|c|c|c|}
\hline \multicolumn{2}{|c|}{ Statistical parameters } & \multicolumn{2}{|c|}{ CoMFA } & \multicolumn{2}{|c|}{ CoMSIA } \\
\hline & & \multicolumn{2}{|c|}{0.511} & \multicolumn{2}{|c|}{0.625} \\
\hline & & \multicolumn{2}{|c|}{5} & \multicolumn{2}{|c|}{1} \\
\hline & & \multicolumn{2}{|c|}{0.822} & \multicolumn{2}{|c|}{0.764} \\
\hline & & \multicolumn{2}{|c|}{0.314} & \multicolumn{2}{|c|}{0.607} \\
\hline & & \multicolumn{2}{|c|}{243.23} & \multicolumn{2}{|c|}{177.36} \\
\hline & & \multicolumn{2}{|c|}{0.590} & \multicolumn{2}{|c|}{0.720} \\
\hline & & \multicolumn{2}{|c|}{0.689} & \multicolumn{2}{|c|}{0.712} \\
\hline & & \multicolumn{2}{|c|}{0.779} & \multicolumn{2}{|c|}{0.781} \\
\hline & & \multicolumn{2}{|c|}{0.052} & \multicolumn{2}{|c|}{0.086} \\
\hline \multicolumn{6}{|l|}{ Contour map } \\
\hline \multirow[b]{2}{*}{ Contour } & \multirow[b]{2}{*}{ Colour } & \multicolumn{2}{|c|}{ CoMFA } & \multicolumn{2}{|c|}{ CoMSIA } \\
\hline & & Contour level & $\begin{array}{l}\text { Volume } \\
\text { estimate }\end{array}$ & Contour level & $\begin{array}{l}\text { Volume } \\
\text { estimate }\end{array}$ \\
\hline \multirow{2}{*}{ Steric } & Yellow & -0.007 & 181.8 & -0.008 & 173.4 \\
\hline & Green & 0.006 & 194.2 & 0.014 & 115.6 \\
\hline \multirow{2}{*}{ Electrostatic } & Red & -0.015 & 89.2 & -0.009 & 35.8 \\
\hline & Blue & 0.020 & 27.6 & 0.019 & 35.1 \\
\hline \multirow{2}{*}{ Hydrophobic } & White & -- & -- & -0.034 & 27.4 \\
\hline & Yellow & -- & -- & 0.023 & 102.2 \\
\hline \multirow{2}{*}{ Donor } & Purple & -- & -- & -0.007 & 64.7 \\
\hline & Cyan & -- & -- & 0.011 & 22.7 \\
\hline \multirow{2}{*}{ Acceptor } & Red & -- & -- & -0.004 & 15.8 \\
\hline & Magenta & -- & -- & 0.003 & 157.5 \\
\hline
\end{tabular}

Note: $\mathrm{N}$ is optimal number of components, $\mathrm{q}^{2}$ is leave-one-out (LOO), $r^{2}$ is non cross-validation coefficient, $r^{2}$ pred is predictive correlation coefficient, SEE is standard error of estimation, $F$ is $F$-test value, $r_{b s}^{2}$ is mean $r^{2}$ of bootstrapping analysis (100 runs), $\mathrm{SD}_{b s}$ is mean standard deviation by bootstrapping analysis. 
of predicted vs observed activity study values used for CoMFA and CoMSIA representations from the training and test set compounds.

\section{CoMFA and CoMSIA contour map analyses}

Final stated non-cross-validated investigation values from CoMFA models were utilized to plot 3D-contour plots of steric and electrostatic fields. Field energies for each lattice point stood well-thought-out as scalar outcomes of the coefficient and standard deviations connected by a precise column of data table (SD*coeff). The map describes the variance in molecular fields linked with the alterations in biological potency of molecules and also specifies the dwelling areas where the associated compounds would positively or negatively connect to the receptor. On the other hand, CoMSIA plot shows the existence of precise functional moiety at a targeted area with that of specified physicochemical property will be preferred or detracted for displaying of decent biological activeness. Interpretation of CoMSIA model was accessible as they specify promising as well as negative interactions towards the receptor with stated parameters like steric, electrostatic, hydrophobic, $\mathrm{H}$-bond donor and acceptor when related to CoMFA representations, since they only display the positive or negative interactions towards receptor through steric and electrostatic counter plots.

CoMFA analysis focusing contour maps displays (steric and electrostatic region) green regions close to methyl of aromatic and outside the aromatic moiety, which suggests that sterically (Figure 11A) large groups remain unaffected our compounds that would decrease the activity. Contour maps of compounds 44 and 1 shows the presence of amide, ethylidine and nitro groups on the $4^{\text {th }}$ position of aromatic moiety related to compound 1 having the carbonyl group and compound $\mathbf{4 4}$ having the nitro atom at the $4^{\text {th }}$ place, which states that presence of electron donating moiety is essential to display the activity. Figures 9 and 10, shows the bulky blue outline around the aromatic and ethylidine moieties show the occurrence of positively charged functional groups that may upsurge the pharmacological activity. Steric contour plots of CoMSIA (Figure 11) are nearly alike to the consequent CoMFA section contour map.

Electrostatic field: Figure 11B reveals that improve in activity due to the occurrence of electron withdrawing groups (red color region) and electron donating groups (blue outlines). Yet, potency of molecule 4 (pMIC $=5.56)$ is because of the existence of heterocyclic pyridazinone moiety.

Hydrophobic field: from CoMSIA model (Figure 11C), it can be reported that yellow regions on the

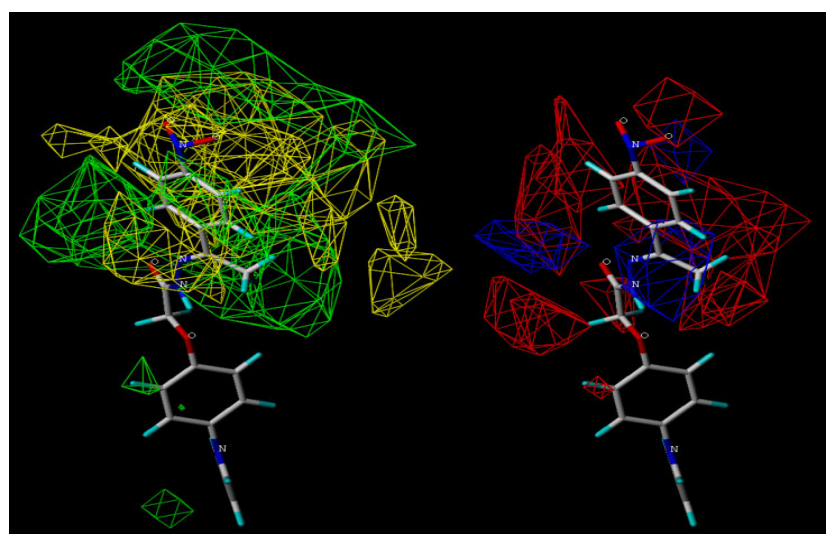

Figure 9: Steric and electrostatic stdev ${ }^{*}$ coef cient map for compound 44 by CoMFA analysis. Sterically favored/unfavored areas are shown in green/yellow contours, while the blue/red contour depicts favorable site for positively/negatively charged groups.

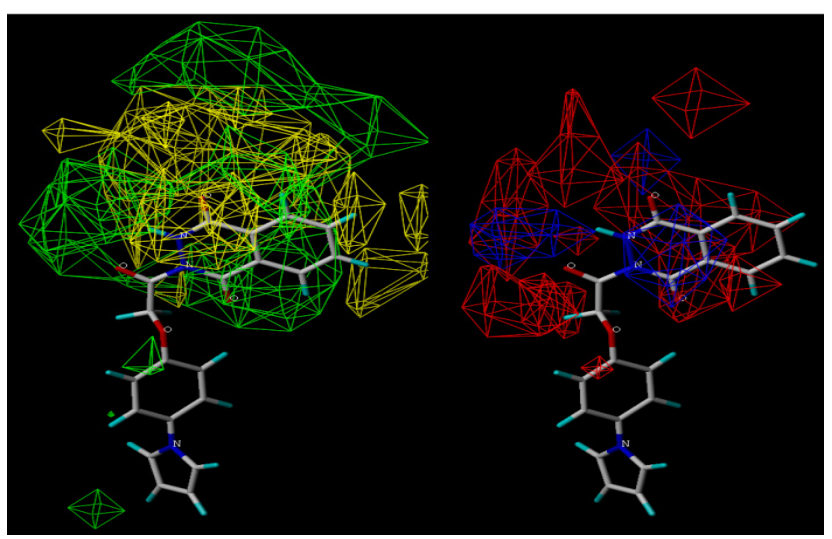

Figure 10: Steric and electrostatic stdev* coef cient map for compound 1 by CoMFA analysis. Sterically favored/unfavored areas are shown in green/yellow contours, while the blue/red contour depicts the favorable site for the positively/negatively charged groups.

aromatic substituents characterizes the promising area for hydrophobic groups, whereas O-atom of -CO, while white counter due to pyrrole ring suggest the preferring consequence of hydrophilic groups.

Hydrogen-bond-acceptor field: Figure 11D, demonstrate the presence of magenta color contour around $\mathrm{C}=\mathrm{O}$ group and protons of the aromatic ring are preferred for desired biological activity.

Hydrogen-bond-donor field: From Figure 11E, the cyan color outline adjacent to the primary amine moiety are most preferred for the antitubercular activity.

\section{Structure-activity relationship study}

As presented in Figure 12, presence of peptide linkage is imperative to display antitubercular activity, if there is the existence of bridge (NH-N---) with bond distance of $2.7 \AA$ and containing a H-bond acceptor through 


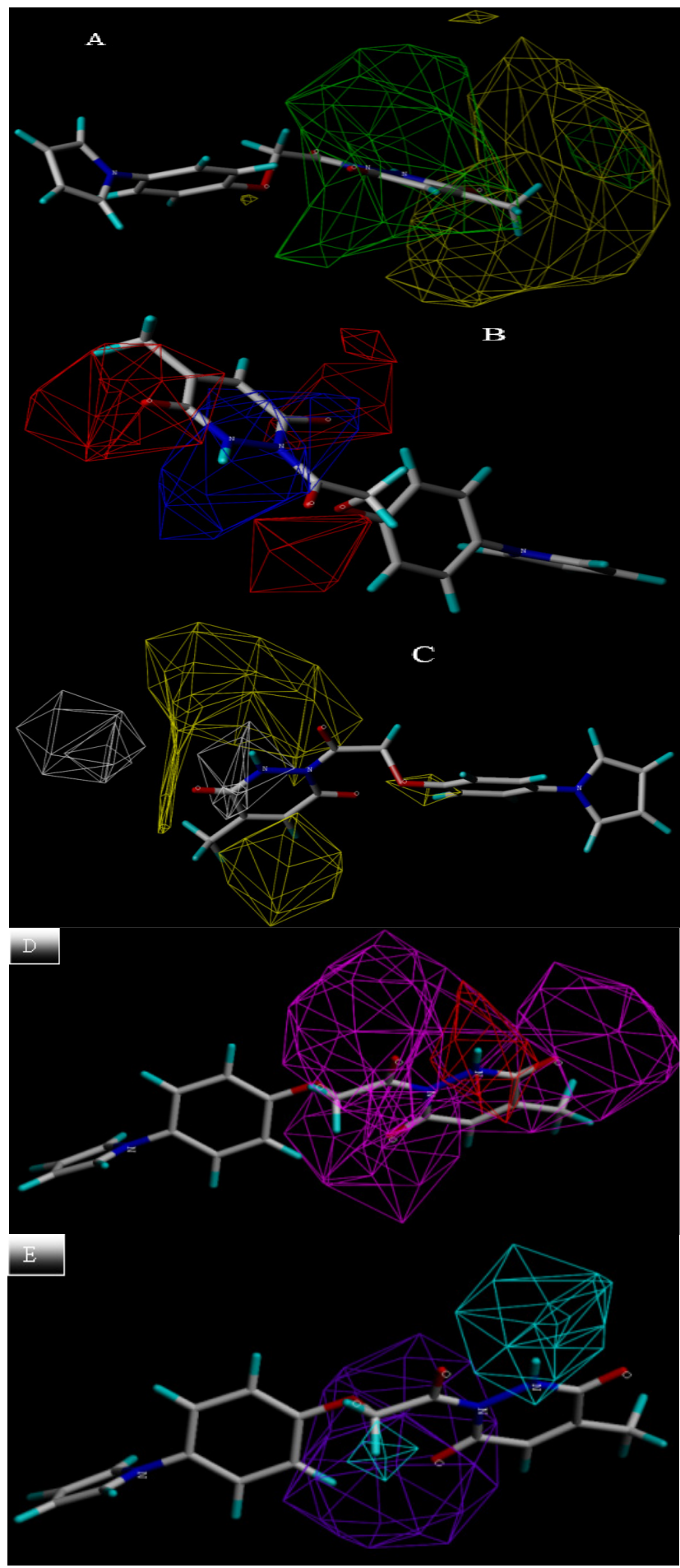

Figure 11: Stdev*Coeff contour maps of CoMSIA analysis for compound 4 by database alignment; (A) Steric contour map. Green and yellow contours refer to sterically favored and disfavored regions, respectively; (B) Electrostatic contour map. Blue and red contours refer to electronic donating and withdrawing groups are favoured region, respectively; (C) Hydrophobic contour map. White contours refer to hydrophilic substituent favored region; (D) Hydrogen bond acceptor contour map. The magenta contours for hydrogen bond acceptor group increase activity, red contours indicate the disfavoured region and (E) Hydrogen bond donor contour map. The cyan and purple contours indicate favorable and unfavorable $\mathrm{H}$ bond donor groups, respectively.

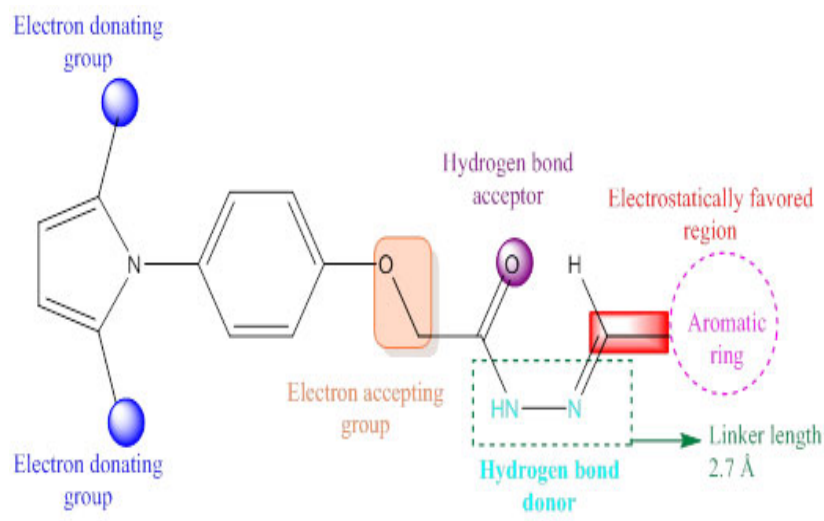

Figure 12: Predicted structure-activity relationship for pyrrolyl derivatives.

electrostatically preferred phenylic moiety, which augments the anti-TB action. Substitution on $2^{\text {nd }}$ and $5^{\text {th }}$ positions of pyrrole aromatic with methyl group is advantageous for antitubercular activity. On the other hand, pyridazinone or aromatic rings are sterically preferred regions and ring substitutions like methyl, methoxy, carbonyl, bromo, chloro and nitro group would upsurge the action.

\section{CONCLUSION}

Docking and 3D-QSAR setup were established for the specified antitubercular action of 53 pyrrole derivatives by means of the statistical packages such as CoMFA and CoMSIA. All the developed models have shown the decent values of statistical and graphical constraints, thereby evidencing favoured likelihood of the created models. Presented study also showed the occurrence of -CO-NH- and aromatic moieties are much needed for unveiling the antitubercular activity. In addition, molecules are in lipophilic environment and hence, are good enough to display pharmacological activities. Stated proto types can be further analysed to design and synthesize the effective novel scaffolds having better antitubercular activities.

\section{ACKNOWLEDGEMENT}

Authors are thankful to Vision Group on Science and Technology, Department of Information Technology, Biotechnology and Science and Technology, Bangalore, India [VGST letter Ref No. VGST/GRD-567/201617/2017-18/183 dated 31-05-2018] for financial support. We thank Dr. H. V. Dambal, President, S. E. T's College of Pharmacy, Dharwad, Karnataka, India for providing necessary facilities. The authors appreciate Dr. Sheshagiri. R. Dixit for his technical support. 


\section{CONFLICT OF INTEREST}

Author's states no conflicts of interest in this work.

\section{REFERENCES}

1. WorldHealthOrganization:http://www.who.int/tb/publications/ globalreport/2018/en/ index.html.1.1.2019

2. Joshi SD, Dixit SR, More UA, Aminabhavi TM, Kulkarni VH, Gadad AK. Enoyl ACP reductase as effective target for the synthesized novel antitubercular drugs: A-state-of-the-art. Mini Rev Med Chem. 2014;14(8):678-93.

3. Hantzsch A, Weber JH. UeberVerbindungen des Thiazols (Pyridins der Thiophenreihe). Ber Dtsch Chem Ges. 1887;20(2):3118-22

4. Cronan JE. Remarkable structural variation within fatty acid mega synthases. Nat Chem Biol. 2006;2(5):232-4.

5. Maier T, Jenni S, Ban N. Architecture of mammalian fatty acid synthase at 4.5 Å resolution. Science. 2006;311(5765):1258-62.

6. Estevez V, Villacampa M, Menendez JC. Multicomponent reactions for the synthesis of pyrroles. Chem Soc Rev. 2010;39(11):402-21.

7. More UA, Joshi SD, Aminabhavi TM, Kulkarni VH, Badiger AM, Lherbet C. Discovery of target based novel pyrrolylphenoxy derivatives as antimycobacterial agents: An in silico approach. Eur $\mathrm{J}$ Med Chem. 2015;94:317-39.

8. Joshi SD, Dixit SR, Kirankumar MN, Aminabhavi TM, Raju KVSN, Narayan R. Synthesis, antimycobacterial screening and ligand-based molecular docking studies on novel pyrrole derivatives bearing pyrazoline, isoxazole and phenyl thiourea moieties. Eur J Med Chem. 2016;107:133-52.

9. Joshi SD, Vagdevi HM, Vaidya VP, Gadaginamath GS. Synthesis of new 4-pyrrol-1-yl benzoic acid hydrazideanalogs and some derived oxadiazole, triazole and pyrrole ring systems: A novel class of potential antibacterial and antitubercular agents. Eur J Med Chem. 2008;43(9):1989-96.

10. Halazy S, Mangus P. Synthesis on the antitumor agent CC-1065: 1-Phenylsulfonyl-1,3-butadiene. An electrophilic equivalent to 1,3-butadiene for the synthesis of 3,31 bipyrroles. Tetrahedron Lett. 1984;25(4):1421-4.

11. Debashish B, Sanghamitra M, Jose C, Grandos JD, Short BK, et al. Ultrasound- assisted bismuth nitrate- induced green synthesis of novel pyrrole derivatives and their biological evaluation as anticancer agents. Eur J Med Chem. 2012;50:209-15.

12. Jones RA, Bean GP. The chemistry of pyrroles. Academics Press, London. 1997.

13. Jones RA. Pyrrole, Part II: The chemistry of heterocyclic compounds. Wiley, New York. 1992
14. Joshi SD, Dixit SR, Basha J, Kulkarni VH, Aminabhavi TM, Nadagouda MN, et al. Pharmacophore mapping, molecular docking, chemical synthesis of some novel pyrrolylbenzamide derivatives and evaluation of their inhibitory activity against enoyl-ACP reductase (InhA) and Mycobacterium tuberculosis. Bio Org Chem. 2018;81:440-53.

15. Joshi SD, Dixit SR, Kulkarni VH, Lherbet C, Nadagouda MN, Aminabhavi TM. Synthesis, biological evaluation and in silico molecular modeling of pyrrolylbenzohydrazides derivatives as enoyl ACP reductase inhibitors. Eur J Med Chem. 2017;126:286-97.

16. Cramer RD, Patterson DE, Bunce JD. Comparative molecular field analysis (CoMFA). 1. Effect of shape on binding of steroids to carrier proteins. J Am Chem Soc. 1988;110(18):5959-67.

17. Klebe $\mathrm{G}$, Abraham $U$, Mietzner T. Molecular similarity indices in a comparative molecular field analysis (CoMFA) of drug molecules to correlate and predict their biological activity. J Med Chem. 1994;37(24):4130-46.

18. Joshi SD, More UA, Dixit SR, Balmi SV, Kulkarni BG, Ullagaddi G, et al. Chemical synthesis and in silico molecular modeling of novel pyrrolybenzohydrazide derivatives: Their biological evaluation against enoylACP reductase (InhA) and Mycobacterium tuberculosis. Bioorg Chem. 2017;75:181-200.

19. Joshi SD, More UA, Panasuriya K, Aminabhavi TM, Gadad AK. Synthesis and molecular modeling studies of novel pyrrole analogues as antimycobacterial agents. J Saudi Chem Soc. 2013;21(1):42-57.

20. Joshi SD, More UA, Aminabhavi TM, Badiger A. Two and three-dimensional QSAR studies on a set of antimycobacterial pyrroles: CoMFA, Topomer CoMFA and HQSAR. Med Chem Res. 2014;23(1):107-26.

21. Joshi SD, More UA, Dixit SR, Korat HH, Aminabhavi TM, Badiger A. Synthesis, characterization, biological activity and 3D-QSAR studies on some novel class of pyrrole derivatives as antitubercular agents. Med Chem Res. 2014;23(3):1123-47.

22. More UA, Joshi SD, Aminabhavi TM, Gadad AK, Nadagouda MN, Kulkarni VH. Design, synthesis, molecular docking and 3D-QSAR studies of potent inhibitors of enoyl-acyl carrier protein reductase as potential antimycobacterial agents. Eur J Med Chem. 2014;17:199-218.

23. Tripos International, Sybyl-X 2.0, Tripos International, St. Louis, MO, USA. 2012.

24. Bush BL, JrNachbar RB. Sample-distance partial least squares: PLS optimized for many variables, with application to CoMFA. J Comput Aided Mol Des. 1993;7(5):587-619.

25. Vong R, Geladi P, Wold S, Svante W, Esbensen K. Source contributions to ambient aerosol calculated by discriminant partial least squares regression. J Chemometr. 1988;2(4):281-96.

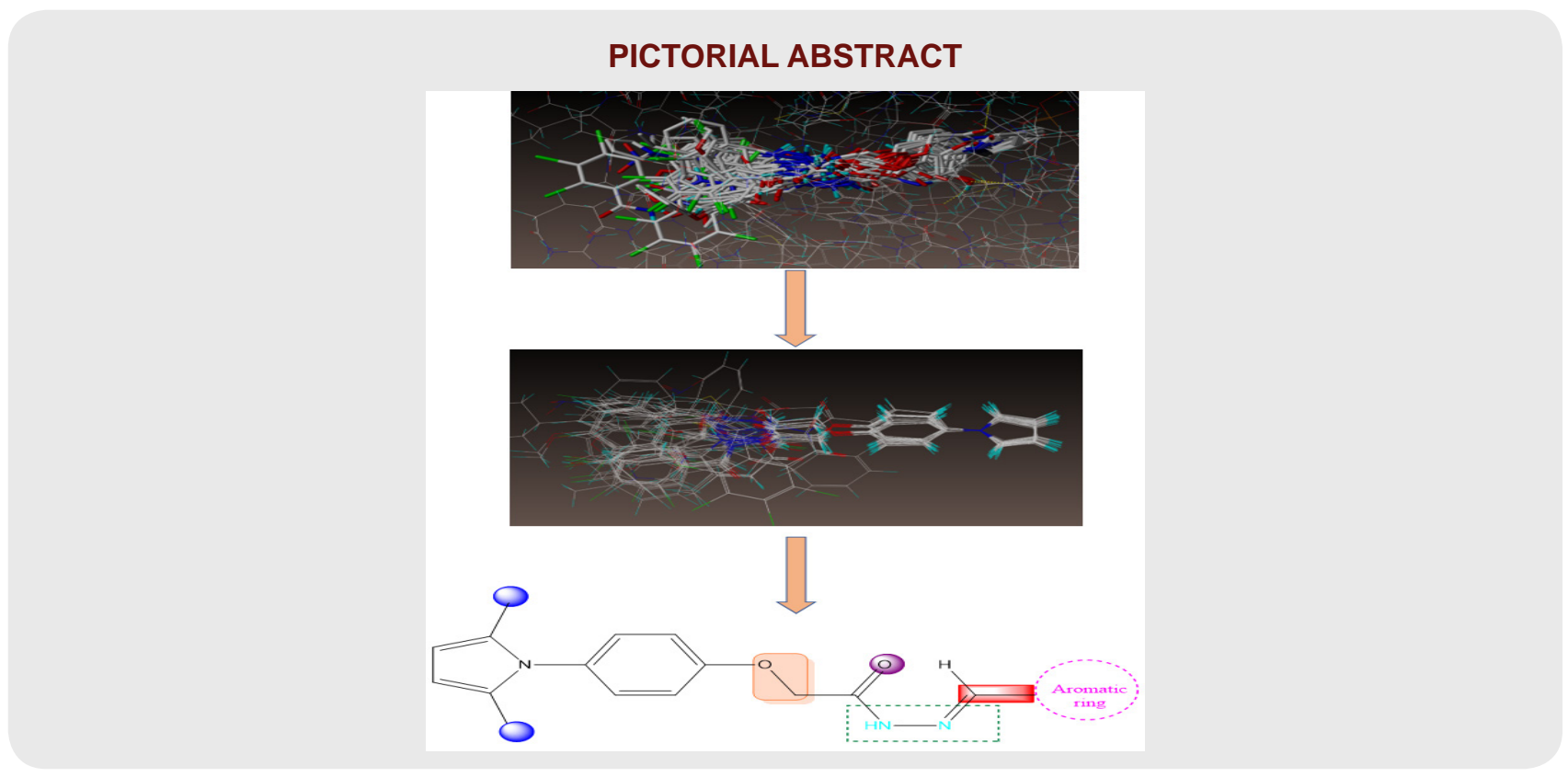




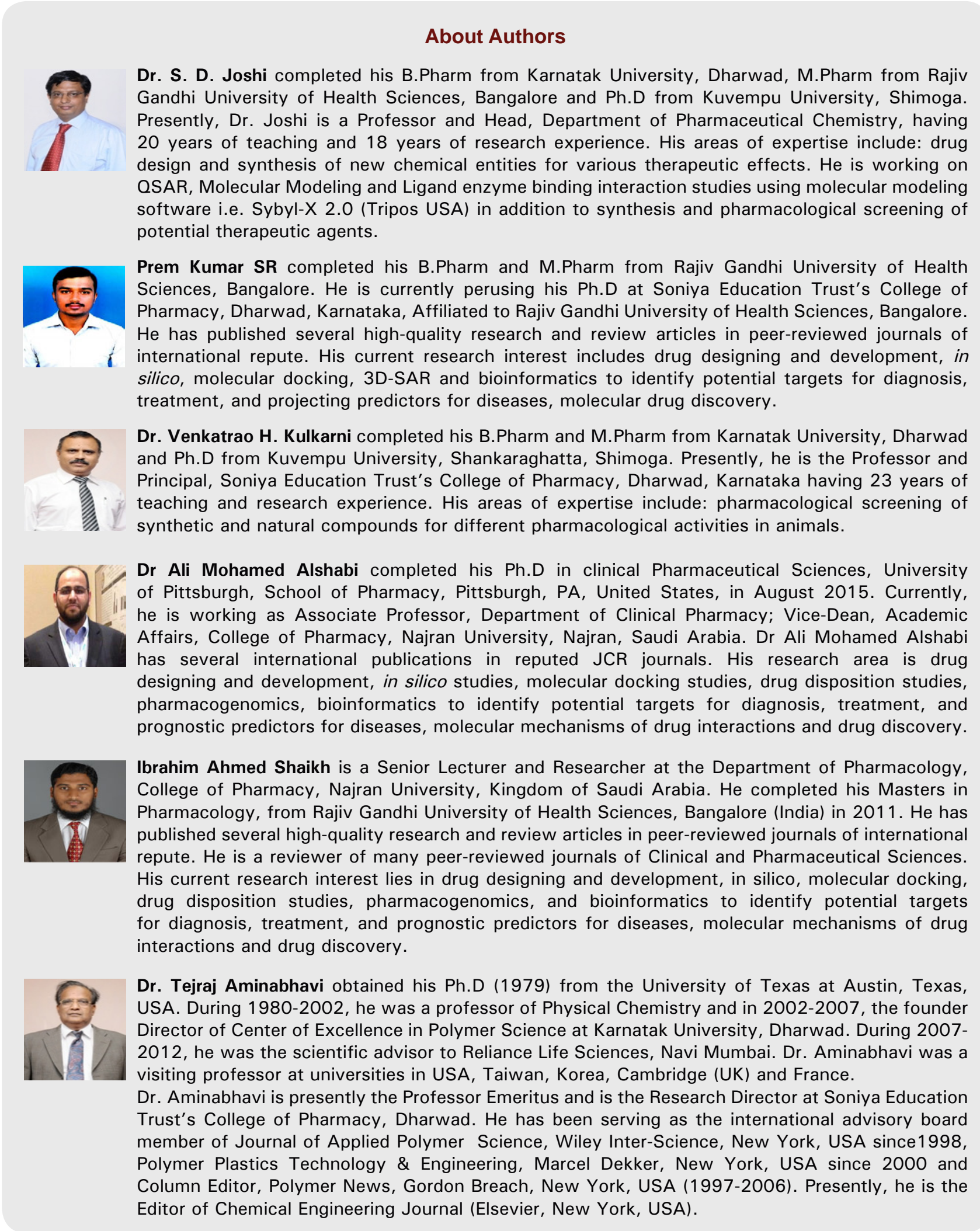

Cite this article: Joshi SD, Kumar SRP, Kulkarni VH, Alshabi AM, Shaikh IA, Aminabhavi TM . In silico docking and 3D-QSAR studies of novel N'-substituted-(pyrrolyl-phenoxy) acetohydrazides as enoyl-ACP reductase antagonists. Indian J of Pharmaceutical Education and Research. 2020;54(3s):s620-s632. 